\title{
Comparación del perfil lipídico y del perfil tiroideo en caninos adultos obesos vs. caninos adultos normales
}

\section{ARTÍCULO DE INVESTIGACIÓN}

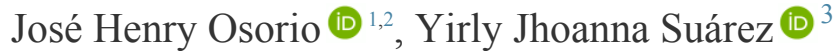 \\ 1 Laboratorio de Investigación en Bioquímica Clínica y Patología Molecular. \\ Departamento de Ciencias Básicas de la Salud, Universidad de Caldas. Manizales, \\ Colombia. \\ 2 Laboratorio de Investigación en Metabolismo, Universidad de Manizales. \\ Manizales, Colombia. \\ 3 Programa Jóvenes Investigadores e Innovadores COLCIENCIAS. Grupo de \\ Investigación: Biosalud, Universidad de Caldas. Manizales, Colombia.
}

jose.osorio_o@ucaldas.edu.co

Recibido: 22 de Noviembre de 2016 y aprobado: 9 de Marzo de 2017, Actualizado: 4 de Septiembre de 2017

DOI: 10.17151/vetzo.2017.11.2.2

RESUMEN: Se estudiaron, en caninos adultos obesos vs. caninos adultos normales, el metabolismo energético y el tiroideo. Se obtuvo suero en ayunas de 80 caninos adultos (40 caninos obesos mayores de un año y 40 caninos normales mayores de un año). Los niveles tirotropina (TSH) y tiroxina libre $\left(\mathrm{T}_{4} \mathrm{~L}\right)$ se determinaron mediante inmunoensayo enzimático; los valores de colesterol total (CT), triacilglicéridos (TAG) y colesterol HDL (C-HDL) por métodos enzimáticoscolorimétricos; los niveles de colesterol LDL (C-LDL) mediante el método directo y los de colesterol VLDL (C-VLDL) con la fórmula de Friedewald (TAG/5). Los valores de promedio de TSH ( $\mu \mathrm{UI} / \mathrm{ml}), \mathrm{T}_{4} \mathrm{~L}(\mathrm{ng} / \mathrm{dl}), \mathrm{CT}(\mathrm{mg} / \mathrm{dl})$, TAG (mg/dl), C-HDL (mg/dl), CVLDL $(\mathrm{mg} / \mathrm{dl})$ y C-LDL $(\mathrm{mg} / \mathrm{dl})$ para los caninos obesos fueron: 1,$04 ; 1,05 ; 242,44$; 58,$82 ; 162,9 ; 11,75 ; 71,68$ respectivamente. Para los caninos adultos normales fueron: 1,$27 ; 0,93 ; 201,49 ; 37,57 ; 134,94 ; 7,42 ; 49,34$ respectivamente. No se encontró diferencia significativa en los niveles de TSH y $\mathrm{T}_{4} \mathrm{~L}$ en caninos adultos obesos vs. caninos adultos normales, (P-valor: 0,51; 0,26 respectivamente). Para todo el perfil lipídico sí se observó diferencia significativa. No se observó diferencia significativa al comparar entre sexos, con una confiabilidad del 95\%. En conclusión, el perfil lipídico se encuentra francamente alterado en los caninos obesos. Con relación al perfil tiroideo no se encuentran diferencias significativas, a pesar de que algunos autores mencionan que el tejido graso incide incrementado los valores en este perfil. 
Palabras clave: metabolismo, canino, tirotropina, tiroxina libre, lípidos.

\title{
Comparison of lipid profile and thyroid profile in obese adult canine vs normal adult canine
}

\begin{abstract}
The energy and thyroid metabolism were studied in obese adult canine vs. normal adult canine. Fasting serum from 80 adult canines was obtained (40 obese canines older than one year and 40 normal canines older than one year). The levels of thyrotropin (TSH) and free thyroxine $\left(\mathrm{T}_{4} \mathrm{~L}\right)$ were determined by enzyme immunoassay. The total cholesterol (TC), triglycerides (TAG) and HDL cholesterol (HDL-C) values were determined by enzymatic-colorimetric methods. The levels of LDL cholesterol (LDL-C) were obtained by the direct method and those of VLDL cholesterol (VLDL-C) were obtained with the Friedewald formula (TAG/5). The mean values of TSH $(\mu \mathrm{UI} / \mathrm{ml}), \mathrm{T}_{4} \mathrm{~L}(\mathrm{ng} / \mathrm{dl}), \mathrm{CT}(\mathrm{mg} / \mathrm{dl})$, TAG $(\mathrm{mg} / \mathrm{dl})$, HDL-C and LDL-C $(\mathrm{mg} / \mathrm{dl})$ for obese adult canines were: $1.04 ; 1.05 ; 242.44 ; 58.82 ; 162.9 ; 11.75 ; 71.68$, respectively. For the normal adult canines, the mean values were: $1.27 ; 0.93 ; 201.49$; $37.57 ; 134.94 ; 7.42 ; 49.34$, respectively. No significant difference was found in the levels of TSH and $\mathrm{T}_{4} \mathrm{~L}$ in adult obese canines vs. normal adult canines (P-value: 0.51; 0.26 , respectively). A significant difference was observed for the lipid profile. No significant difference was observed when comparing male/female canines with $95 \%$ reliability. In conclusion, the lipid profile is clearly altered in obese canines. Regarding the thyroid profile, no significant differences were found, although some author mention that fatty tissue affects this profile.
\end{abstract}

Key words: metabolism, canine, thyrotropin, free thyroxine, lipids.

\section{Introducción}

La obesidad es la enfermedad nutricional más frecuente en los perros y se define como una acumulación excesiva de tejido adiposo (Burkholder \& Toll, 2000). Cerca del 40\% de los perros que son llevados al veterinario sufren de obesidad (Pibot et al., 2008), siendo esta poco percibida por los propietarios ya que los motivos de consulta son totalmente ajenos a este problema. Dicho desorden se presenta cuando la ingesta de energía excede constantemente el gasto energético diario. Normalmente las concentraciones de grasa corporal se encuentran entre $10-20 \%$ en animales con peso adecuado, considerándose un inicio de obesidad en la mascota cuando presenta un $10 \%$ de sobrepeso y cuando alcanza el $20 \%$ ya es considerada una obesidad severa (Zentek et al., 2010). Es importante detectar y controlar la obesidad, ya que los perros obesos tienen más riesgo de padecer problemas de salud como: enfermedades ortopédicas traumáticas y degenerativas, enfermedades cardiovasculares, enfermedades dermatológicas, distocias, intolerancia al calor y al ejercicio; predisposición a diabetes mellitus, hipertensión, hiperlipidemia, disminución de la respuesta inmune y aumento 
del riesgo en anestesia (Fettman et al., 1997; Burkholder, 2001; Kuruvilla \& Frankel, 2003).

En humanos y ratas se ha demostrado que las hormonas tiroideas influyen directamente en la homeostasis de la glucosa. El hipotiroidismo se asocia con alteraciones en las vías metabólicas debido al papel central de la hormona tiroidea en el metabolismo intermedio. La obesidad y la hiperlipidemia son dos características principales del canino hipotiroideo, las cuales se han asociado con hiperinsulinemia en los seres humanos y los perros (Kahn \& Flier, 2000; Lewis et al., 2002). Una deficiencia de la hormona tiroidea puede llevar directamente a una insulinorresistencia. Además, las hormonas tiroideas modulan la actividad de algunas enzimas reguladoras del metabolismo de la glucosa (Rochon et al., 2003).

Los pacientes con disfunción tiroidea presentan alteraciones metabólicas, es por eso que los pacientes con hipotiroidismo se caracterizan por tener una tasa metabólica baja, lipólisis reducida, niveles de triglicéridos y colesterol elevados. En cambio, los pacientes con hipertiroidismo padecen alteraciones en el metabolismo de los carbohidratos, resistencia a la insulina, pérdida significativa de peso asociada principalmente a reducción de grasa y masa muscular (López-Pompey, 2012).

\section{Materiales y Métodos}

Este estudio fue de casos y controles. Se tomaron en estado de ayuno muestras de sangre de 80 caninos criollos o sus cruces del departamento de Caldas, Colombia

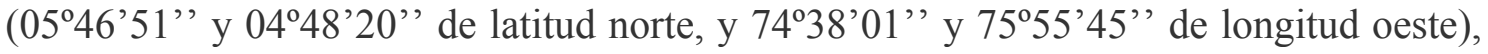
donde 40 caninos obesos mayores de un año fueron catalogados como grupo caso y 40 caninos mayores de un año con condición corporal óptima como grupo control. Ambos grupos fueron diferenciados por género (20 hembras y 20 machos). La clasificación de la condición corporal de los animales se realizó según los criterios realizados por Tams (2004). Los animales estaban clínicamente sanos, no se tuvieron en cuenta hembras en gestación ni en lactancia, ni animales que recibieran medicamentos que pudieran alterar el metabolismo lipídico o el tiroideo. La toma de las muestras de sangre se realizó en las horas de la mañana, con vacutainer sin anticoagulante mediante venopunción en la yugular. Se refrigeraron las muestras mientras se llevaban al laboratorio. Luego, se centrifugaron a $3500 \mathrm{rpm}$ en una centrífuga (Thermo de serie IEC CL31 Multispeed) durante 15 min. Posteriormente, fue extraído el suero, y se conservó a $-30^{\circ} \mathrm{C}$ hasta su posterior análisis. 


\section{Medición del perfil lipídico}

De acuerdo a los resultados obtenidos en estudios hechos en especies como los caninos con patrón metabólico HDL para el metabolismo de los lípidos (Osorio et al., 2012a, Osorio et al., 2012b), los valores de colesterol total, triacilglicéridos y colesterol HDL fueron determinados por métodos enzimáticos-colorimétricos, de acuerdo a las recomendaciones del fabricante. Los análisis de los resultados se realizaron en un analizador semiautomático de química Rayto RT-1904C (Rayto Life and Analytical Sciences Co., Ltd. Shenzhen, China).

Colesterol total (CT). La determinación del CT en suero se efectúo mezclando $10 \mu \mathrm{L}$ de la muestra y $1 \mathrm{ml}$ de reactivo (Pipes $35 \mathrm{mmol} / \mathrm{L}$, colato sódico $0,5 \mathrm{mmol} / \mathrm{L}$, fenol 28 $\mathrm{mmol} / \mathrm{L}$, colesterol esterasa $>0,2 \mathrm{U} / \mathrm{ml}$, colesterol oxidasa $>0,1 \mathrm{U} / \mathrm{ml}$, peroxidasa $>0,8$ $\mathrm{U} / \mathrm{ml}$, 4-aminoantipirina (4-AA) $0,5 \mathrm{mmol} / \mathrm{L}, \mathrm{pH} 7,0$ ). Se agitó manualmente la mezcla y se dejaron incubar los tubos durante $10 \mathrm{~min}$ a temperatura ambiente. Los ésteres de colesterol se hidrolizan por la colesterol esterasa y dan lugar a colesterol libre, el cual por acción de la colesterol oxidasa forma colestenona + peróxido de hidrógeno, este último en presencia de la 4-AA y fenol da lugar a la quinonaimina por acción de la peroxidasa. La quinonaimina es proporcional al CT de la muestra y se cuantifica espectrofotométricamente (analizador semiautomático de química, RAYTO RT-1904C, Rayto Life and Analytical Sciences Co., Ltd. Shenzhen, China), a una longitud de onda de $500 \mathrm{~nm}$.

Triacilglicéridos (TAG). Se determinaron los TAG en suero, utilizando $10 \mu \mathrm{L}$ de la muestra y $1 \mathrm{ml}$ de reactivo (Pipes $45 \mathrm{mmol} / \mathrm{L}$, 4-clorofenol $6 \mathrm{mmol} / \mathrm{L}$, cloruro magnésico $5 \mathrm{mmol} / \mathrm{L}$, lipasa $>100 \mathrm{U} / \mathrm{ml}$, glicerol-quinasa $>1,5 \mathrm{U} / \mathrm{ml}$, glicerol-3Poxidasa $>4 \mathrm{U} / \mathrm{ml}$, peroxidasa $>0,8 \mathrm{U} / \mathrm{ml}, 4-\mathrm{AA} 0,75 \mathrm{mmol} / \mathrm{L}$, ATP 0,9 mmol/L, pH 7,0). Se agitó manualmente la mezcla y se dejaron incubar los tubos durante $15 \mathrm{~min}$ a temperatura ambiente. Con este proceso, los TAG son hidrolizados por la lipasa hasta glicerol y ácidos grasos; el glicerol, en presencia de Adenosín Trifosfato (ATP), es fosforilado por la glicerol-quinasa y da lugar al glicerol 3P + ADP; el glicerol 3P en presencia de oxígeno forma peróxido de hidrógeno por acción de la glicerol-3P-oxidasa. Finalmente, se cuantificó espectrofotométricamente (analizador semiautomático de química, RAYTO RT-1904C, Rayto Life and Analytical Sciences Co., Ltd. Shenzhen, China), a una longitud de onda de $500 \mathrm{~nm}$, la quinonaimina producto de la acción de la peroxidasa sobre el peróxido de hidrógeno en presencia de 4-AA y clorofenol.

Determinación del colesterol HDL mediante el método de precipitación con fosfotungstato. Para aplicar este método se usó $1 \mathrm{ml}$ de reactivo (fosfotungstato 0,4 $\mathrm{mmol} / \mathrm{L}$ y cloruro de magnesio $20 \mathrm{mmol} / \mathrm{L}$ ), que se mezcló con $0,2 \mathrm{ml}$ de la muestra de suero, se agitó manualmente y se dejó durante 10 min a temperatura ambiente, se centrifugó por $10 \mathrm{~min}$ a $4000 \mathrm{rpm}$ (Thermo Scientific IEC CL40R Refrigerated Benchtop Centrifuge, 1210927, Alemania). En el precipitado se sedimentan las VLDL, las lipoproteínas de densidad intermedia (IDL, del inglés Intermediate Density 
Lipoprotein) y LDL, y en el sobrenadante permanecen las HDL. Se toman con cuidado $100 \mu \mathrm{L}$ del sobrenadante, y se mezclan en otro tubo con $1 \mathrm{ml}$ del reactivo para CT y se incuban por 10 min al baño maría (Memmert WB350 waterbath, Alemania) a $37^{\circ} \mathrm{C}$. El C-HDL es hidrolizado por la colesterol esterasa y la colesterol oxidasa, dando lugar a peróxido de hidrógeno que es consumido por una peroxidasa en presencia de la 4-AA y fenol, quedando como producto final la quinonaimina, siendo este producto proporcional al C-HDL de la muestra, luego se cuantifica espectrofotométricamente (analizador semiautomático de química, RAYTO RT-1904C, Rayto Life and Analytical Sciences Co., Ltd. Shenzhen, China) a una longitud de onda de $500 \mathrm{~nm}$.

Determinación del colesterol LDL mediante el método directo. Un detergente específico solubiliza el colesterol de las lipoproteínas de alta densidad (HDL), las de muy baja densidad (VLDL) y los quilomicrones. Los ésteres de colesterol son hidrolizados por la colesterol esterasa y la colesterol oxidasa mediante una reacción no formadora de color. Un segundo detergente solubiliza el colesterol de las lipoproteínas de baja densidad (LDL) de la muestra, y el colesterol LDL se cuantifica espectrofotométricamente.

Colesterol VLDL. Los niveles de colesterol VLDL fueron determinados dividiendo los niveles de los triglicéridos entre 5 (TAG/5).

\section{Medición del perfil tiroideo}

Para la determinación del T4 libre se utilizó la prueba de inmunoensayo enzimático competitivo (Accubind $\mathrm{T}_{4} \mathrm{~L}$, MonobindInc $\mathrm{C}$ ), donde los sueros de los caninos normales y obesos son colocados en contacto con una fase sólida que contiene anticuerpos contra la T4, a la cual se le agrega el conjugado compuesto por T4 libre unido a peroxidasa de rábano (HRP). Luego de $1 \mathrm{~h}$ de incubación a temperatura ambiente, se hace un lavado para liberar aquellas moléculas no unidas y se agrega el substrato formado por una mezcla de tetrametil bencidina (TMB) y peróxido de hidrógeno disuelto en buffer de acetato, con una incubación de $15 \mathrm{~min}$, tiempo al cabo del cual se detiene la reacción al agregarle una solución de ácido clorhídrico 1N. La lectura se hace en un equipo lector de microELISA (Titertek Multiscan ${ }^{\mathrm{TM}}$ ) a una absorbancia de $450 \mathrm{~nm}$. Las absorbancias obtenidas de los estándares se grafican junto con las concentraciones, y de la curva de calibración se obtienen las concentraciones de T4 libre de las respectivas muestras. Para la determinación de los niveles de TSH (hormona estimulante del tiroides), se utiliza una prueba de inmunoensayo enzimático colorimétrico tipo sándwich, utilizada para la cuantificación del TSH de origen humano (Accubind TSH-MonobindInc®). A una placa de 96 pozos, que tiene unidos anticuerpos monoclonales contra la TSH en una interacción estreptavidina-biotina, se le agrega una alícuota de suero obtenido de caninos adultos y una alícuota de conjugado compuesta de un anticuerpo policlonal contra la TSH unido a la peroxidasa de rábano. Se incuba dos h a temperatura ambiente, tiempo al cabo del cual se lava la placa con una solución de fosfatos para eliminar todas aquellas moléculas no unidas, y se agrega el substrato formado por una mezcla de 
tetrametil bencidina (TMB) y de peróxido de hidrógeno. Gracias a la presencia de la enzima en el complejo inmune previamente formado, se produce la generación de oxígeno a partir de peróxido de hidrógeno, el cual al actuar sobre la tetrametil bencidina genera un cambio de color cuya intensidad es proporcional a la concentración de la hormona. Para detener la reacción enzimática luego de un periodo de incubación de 15 min se agrega ácido clorhídrico $1 \mathrm{~N}$, y posteriormente se procede a hacer la lectura en un fotómetro lector de microELISA (Titertek Multiscan ${ }^{\mathrm{TM}}$ ) a una longitud de onda de 450 $\mathrm{nm}$. Los resultados obtenidos de los estándares se grafican frente a sus concentraciones, generándose una curva de calibración en la cual se pueden obtener las concentraciones de cada uno de los sueros probados.

\section{Análisis estadístico}

Se evaluaron las diferencias sobre los distintos grupos por medio de un análisis de varianza usando el programa Stata versión 13 para Windows, donde se aceptaba diferencia estadísticamente significativa cuando $\mathrm{P}<0,05$.

\section{Resultados}

Al comparar de forma general los caninos normales con los caninos obesos con respecto al perfil lipídico ( $\mathrm{mg} / \mathrm{dl})$, se pudo evidenciar que existe diferencia significativa en todas las variables con un nivel de confianza del 95\%, ya que el P-valor fue menor a 0,05 siendo más altos los valores en los caninos obesos (Tabla 1). Específicamente, cuando comparamos machos normales con machos obesos hallamos diferencia en el colesterol HDL $(\mathrm{P}=0,004)$ y colesterol LDL $(\mathrm{P}=0,005)$ (Tabla 2$)$, y cuando comparamos hembras normales $\mathrm{y}$ hembras obesas se pueden encontrar diferencias en colesterol total $(\mathrm{P}=0,030)$, triglicéridos $(\mathrm{P}=0,006)$, colesterol HDL $(\mathrm{P}=0,027)$, colesterol VLDL $(\mathrm{P}=0,006)$, colesterol LDL $(0,034)$ (Tabla 3$)$.

En el perfil tiroideo no se encontró diferencia significativa en el grupo de los normales y en el grupo de los obesos cuando se discrimina entre sexo, igualmente no se encontró diferencia significativa con un nivel de confianza del 95\% cuando se comparan caninos adultos obesos con caninos adultos normales para $\mathrm{TSH}(\mu \mathrm{UI} / \mathrm{ml})(\mathrm{P}=0,51)$ y para $\mathrm{T} 4$ Libre (ng/dl) $(\mathrm{P}=0,26)($ Tablas 4, 5 y 6). 
Tabla 1. Niveles de perfil lipidico en caninos normales y caninos obesos

\begin{tabular}{ccccccccccc}
\hline Perfil & \multicolumn{4}{c}{ Caninos normales } & \multicolumn{4}{c}{ Caninos obesos } & P-valor \\
\cline { 2 - 8 } lipídico & $\mathbf{X}$ & De & Min & Max & $\mathbf{X}$ & De & Min & Max & \\
CT & 201,49 & 48,35 & 126,22 & 337,37 & 242,44 & 78,36 & 125,86 & 337,35 & $\underline{0,008^{*}}$ \\
TAG & 37,57 & 22,95 & 11,73 & 111,43 & 58,82 & 47,05 & 6,83 & 253,20 & $\underline{0,017^{*}}$ \\
C-HDL & 134,94 & 33,23 & 75,20 & 223,74 & 162,9 & 36,45 & 82,73 & 252,23 & $\underline{0,000^{*}}$ \\
C-VLDL & 7,42 & 4,58 & 2,34 & 22,25 & 11,75 & 9,40 & 1,34 & 50,61 & $\underline{0,017^{*}}$ \\
C-LDL & 49,34 & 18,68 & 24,18 & 95,95 & 71,68 & 32,14 & 22,71 & 170,89 & $\underline{0.000^{*}}$ \\
\hline
\end{tabular}

CT: colesterol total. TAG: triglicéridos. C-HDL: colesterol HDL. C-VLDL: colesterol VLDL.

C-LDL: colesterol LDL. De: Desvisción estándar. Min: mínimo. Max: máximo. "Indics diferencis significativa $P$-valor $<0,05$.

Tabla 2. Niveles de perfil lipidico en caninos machos normales y caninos machos obesos

\begin{tabular}{|c|c|c|c|c|c|c|c|c|c|}
\hline Perfil & & Macho & ormales & & & Macho & obesos & & P-valor \\
\hline lipídico & $x$ & $\mathrm{De}$ & Min & $\operatorname{Max}$ & $x$ & $\mathrm{De}$ & Min & $\operatorname{Max}$ & \\
\hline $\mathrm{CT}$ & 187,6 & 41,81 & 127,07 & 275,37 & 217 & 62,54 & 125,84 & 333,53 & 0,110 \\
\hline TAG & 37,30 & 28,29 & 13,49 & 111,43 & 45,60 & 32,50 & 6,80 & 126,60 & 0.439 \\
\hline C-HDL & 121.93 & 28,01 & 75,20 & 189,29 & 152,20 & 37,39 & 82,73 & 224,51 & $\underline{0,004^{*}}$ \\
\hline C-VLDL & 7,44 & 5,63 & 2,68 & 22,27 & 9,10 & 6,49 & 1,32 & 25,31 & 0.439 \\
\hline C-LDL & 45,34 & 18,84 & 25,80 & 95,19 & 65,49 & 26,20 & 22,72 & 117,50 & $\underline{0,005^{*}}$ \\
\hline
\end{tabular}

CT: colesterol total. TAG: triglicéridos. C-HDL: colesterol HDL. C-VLDL: colesterol VLDL. C-LDL: colesterol LDL. De: Desviación estándar. Min: mínimo. Max: máximo. "Indica diferencia significativa P-valor<0, 05 .

Tabla 3. Niveles de perfil lipidico en caninos hembras normales y caninos hembras obesss

\begin{tabular}{cccccccccc}
\hline Perfil & \multicolumn{3}{c}{ Hembras normales } & \multicolumn{3}{c}{ Hembras obesas } & P-valor \\
\cline { 2 - 8 } lipidico & $\mathbf{X}$ & De & Min & Max & $\mathbf{X}$ & De & Min & Max & \\
CT & 217,65 & 51,40 & 126,21 & 337,36 & 268,03 & 85,54 & 175,89 & 486,27 & $\underline{0,030^{*}}$ \\
TAG & 37,89 & 15,49 & 11,74 & 73,66 & 72,21 & 55,83 & 18,69 & 253,22 & $\underline{0,006^{*}}$ \\
C-HDL & 149,88 & 33,01 & 88,69 & 223,73 & 173,84 & 32,89 & 125,15 & 252,23 & $\underline{0,027^{*}}$ \\
C-VLDL & 7,49 & 3,99 & 2,33 & 14,71 & 14,42 & 11,15 & 3,73 & 50,62 & $\underline{0,006^{*}}$ \\
C-LDL & 54,32 & 17,70 & 24,17 & 95,95 & 77,89 & 36,79 & 31,49 & 170,89 & $\underline{0,034^{*}}$ \\
\hline
\end{tabular}

CT: colesterol total. TAG: triglicéridos. C-HDL: colesterol HDL. C-VLDL: colesterol VLDL. C-LDL: colesterol LDL. De: Desviación estándar. Min: mínimo. Max: máximo. "Indica diferencia significativa P-valor $<0,05$.

Tabla 4. Comparación de perfil tiroideo entre caninos adultos normales vs. caninos sdultos obesos

\begin{tabular}{cccccccccc}
\hline Perfil & \multicolumn{3}{c}{ Caninos normales } & \multicolumn{4}{c}{ Caninos obesos } & P-valor \\
\cline { 2 - 8 } tiroideo & $\mathbf{X}$ & De & Min & Max & $\mathbf{X}$ & De & Min & Max & \\
TSH & 1,27 & 2,74 & 0,9 & 17,65 & 1,04 & 1,62 & 0,9 & 9,35 & 0,518 \\
T4L & 0,93 & 0,39 & 0,31 & 2,15 & 1,05 & 0,43 & 0,21 & 1,95 & 0,262 \\
\hline
\end{tabular}


Tabla 5. Niveles de perfil tiroideo en caninos machos

\begin{tabular}{cccccccccc}
\hline Perfil & \multicolumn{4}{c}{ Machos normales } & \multicolumn{4}{c}{ Machos obesos } & P-valor \\
\cline { 2 - 8 } tiroideo & $\mathbf{X}$ & De & Min & Max & $\mathbf{X}$ & De & Min & Max & \\
TSH & 0,49 & 0,12 & 0,1 & 0,72 & 1,15 & 2,14 & 0,10 & 9,35 & 0,623 \\
T4L & 0,90 & 0,36 & 0,31 & 2,15 & 0,99 & 0,43 & 0,21 & 1,95 & 0,677 \\
\hline
\end{tabular}

\begin{tabular}{|c|c|c|c|c|c|c|c|c|c|}
\hline \multirow{2}{*}{$\begin{array}{l}\text { Perfil } \\
\text { tiroideo }\end{array}$} & \multicolumn{4}{|c|}{ Hembras normales } & \multicolumn{4}{|c|}{ Hembras obesas } & \multirow[t]{2}{*}{ P-valor } \\
\hline & $x$ & $\mathrm{De}$ & Min & $\operatorname{Max}$ & $x$ & $\mathrm{De}$ & Min & $\operatorname{Max}$ & \\
\hline TSH & 2,15 & 3,93 & 0,1 & 17,4 & 1,20 & 1,03 & 0,1 & 3,39 & 0,210 \\
\hline T4L & 1,0 & 0,48 & 0,35 & 2,05 & 1,2 & 0,43 & 0,53 & 1,95 & 0.256 \\
\hline
\end{tabular}

\section{Discusión}

Estudios anteriores comparaban el perfil lipídico entre caninos normales vs. caninos obesos. Sin embargo, no se comparaba entre sexos. En una investigación se reportó una diferencia significativa entre el colesterol LDL, siendo los caninos obesos quienes presentan los valores más elevados, sin embargo se pudo observar que los niveles de colesterol total, triglicéridos y colesterol VLDL eran más altos en los caninos obesos, y a diferencia del presente estudio el colesterol HDL es menor en el grupo de caninos obesos (Osorio \& Giraldo, 1999).

Otros autores reportan que los niveles de triglicéridos y colesterol total, son más altos en los animales obesos, sin embargo no se reportan diferencias estadísticamente significativas (Veiga et al., 2008; Eirmann et al., 2009). Mientras que otros, han encontrado que los niveles de colesterol total y triglicéridos son más elevados en perros obesos, encontrando una diferencia significativa entre los valores de ambos grupos (Peña et al., 2008).

En niños se ha evidenciado que el colesterol total, triglicéridos y colesterol-VLDL fueron significativamente mayores en niños y adolescentes obesos cuando son comparados con no obesos (Rojas-Gabulli et al., 2010; Samaan, 2012). En el presente estudio, no se encontró diferencia significativa en el perfil tiroideo cuando se comparan caninos normales con caninos obesos, sin embargo estudios hablan de que existen factores como la obesidad que hacen que en los perros eutiroideos obesos presenten incrementos en los valores de T3 y T4 plasmáticos (Marca et al., 1996).

En seres humanos se ha evaluado la relación existente entre la adiposidad del obeso eutiroideo y los niveles séricos elevados de TSH, encontrando una significativa 
correlación entre los niveles de TSH en suero con el peso corporal (Mehmet et al., 2007; Samaan, 2012). Igualmente, en niños obesos se encontraron niveles elevados de TSH y una correlación significativa de esta con el índice de masa corporal y los niveles de insulina (Dekelbab et al., 2010). Se debe tener en cuenta que los valores del perfil lipídico (Osorio \& Giraldo, 1999; Osorio et al., 2012a) y del perfil tiroideo (Ramsey et al., 1997; Melián-Limiñana et al., 1999; Matamoros et al., 2002; Ramírez-Benavides \& Osorio, 2009) pueden ser más bajos que los de otros estudios, y esto se deba posiblemente a la utilización de diferentes técnicas o de diferentes kits para la determinación de cada una de las variables estudiadas.

\section{Conclusión}

En conclusión, el perfil lipídico se encuentra francamente alterado en los caninos obesos coincidiendo con lo reportado. Sin embargo, de manera implícita, se puede evidenciar de un factor de protección cardiovascular, ya que el colesterol HDL es más alto en ellos. En cuanto al perfil tiroideo, no se encontraron diferencias significativas, discrepando de otros trabajos donde el tejido graso incide en los valores de T3 y T4.

\section{Agradecimientos}

A Jorge Enrique Pérez del Laboratorio de Microbiología de la Universidad de Caldas por la asesoría en la medición de las muestras.

\section{Referencias bibliográficas}

- Burkholder, W.J. Precision and practicality of methods assessing body composition of dogs and cats. Comp Cont Educ Pract, v.23, p.1-10, 2001.

- Burkholder, W.J.; Toll, P.W. Obesity. In: Hand, M.S.; Thatcher, C.D.; Remillard, R.L.; Roudebush, P. (Ed). Small Animal Clinical Nutrition. Marceline, MO: Walsworth Publishing Co., 2000. p.404-406.

- Dekelbab, B.H.; Abou Ouf, H.A.; Jain, I. Prevalence of Elevated Thyroid-Stimulating hormone levels in obese children and adolescents. Endocrine Practice, v.16, n.2, p.187-190, 2010. 
- Eirmann, L.A.; Freeman, L.M.; Laflamme, D.P. et al. Comparativa de adipokine concentraciones y los marcadores de inflamación en pacientes obesos versus perros de lean. Intern J Appl Res Vet Med, v.7, n.4, p.196-205, 2009.

- Fettman, M.J.; Stanton, C.A.; Banks, L.L. et al. Effects of neutering on bodyweight, metabolic rate and glucose tolerance in domestic cats. Res Vet ScienK, v.62, p.131136, 1997.

- Kahn, B.B.; Flier, J.S. Obesity and insulin resistance. The Journal of Clinical Investigation, v.106, p.473-481, 2000.

- Kuruvilla, A.; Frankel, T.L. Heart rate of pet dogs: effects of overweight and exercise.Asia Pacifi c J Clin Nutr, v.12, p.51, 2003.

- Lewis, G.F.; Carpentier, A.; Adeli, K. Disordered fat storage and mobilization in the pathogenesis of insulin resistance and type 2 diabetes. Endocrine Reviews, v.23, p.201229, 2002.

- López-Pompey, N.A. Asociación entre el estado de la función tiroidea, parámetros metabólicos, niveles de anticuerpos anti tiroperoxidasa y los niveles séricos de la Adipocitoquina chemerin. Bogotá, Colombia: Universidad Nacional de Colombia, 2012. Tesis (Especialista en Endocrinología).

- Marca, M.C.; Loste, A.; Sanz, M.C. et al. Hipotirodismo canino: Revision y actualización de su diagnostic. Avepal, v.16, n.3, p.111-117, 1996.

- Matamoros, R.; Gómez, C.; Andaur, M. Hormonas de utilidad diagnóstica en Medicina Veterinaria. Arch med vet, v.34, n.2, p.167-182, 2002.

- Mehmet, B.; Fulya, A.; Esma, A. et al. Obesity is associated with increased serum TSH level, independent of thyroid function. Swiss Med Wkly, v.137, p.431-434, 2007.

- Melián-Limiñana, C.; Morales-Doreste, M.; Pérez-Alenza, M.D. Concentración de TSH endógena: un nuevo test para el diagnóstico de hipotiroidismo canino. Clínica Veterinaria de Pequeños Animales (Avepa), v.19, n.2, p.71-75, 1999.

- Osorio, J.H.; Giraldo, C.E. Perfil lipídico en caninos adultos obesos vs caninos adultos normales. Revista Veterinaria y Zootecnia de Caldas, v.11, n.1, p.7-10, 1999.

- Osorio, J.H.; Suárez, Y.J.; Pérez, J.E. Estudio del perfil lipídico canino por edad y sexo. Rev Med Vet, v.23, p.65-72, 2012a.

- Osorio, J.H.; Vinazco, J.; Pérez, J.E. Comparación de perfil lipídico por sexo y edad en bovinos. Biosalud, v.11, n.1, p.25-33, $2012 \mathrm{~b}$.

- Peña, C.; Suárez, L.; Bautista, I. et al. Relationship between analytic values and canine obesity. J Anim Physiol Anim Nutr (Berl), v.92, n.3, p.324-325, 2008. 
- Pibot, P.; Biourge, V.; Elliott, D. Enciclopedia de la nutrición clínica canina. Royal Canin. Paris: Aniwa Pub, 2008, p.509.

- Ramírez-Benavides, G.F.; Osorio, J.H. Niveles séricos de Tetrayodotironina libre (T4L), Mediante el método de electroquimioluminiscencia en caninos. RC FCV-LUZ, v.19, n.3, p.238-241, 2009.

- Ramsey, L.K.; Evans, H.; Herrtage, M.E. Thyroid stimulating hormone and total thyroxine concentrations in euthyroid, sick euthyroid and hypothyroid dogs. J Small Anim Pract, v.38, p.540-545, 1997.

- Rochon, C.; Tauveron, I.; Dejax, C. et al. Response of glucose disposal to hyperinsulinaemia in human hypothyroidism and hyperthyroidism. Clinical Science, v.104, p.7-15, 2003.

- Rojas-Gabulli, M.I.; Núñez, O.; Del Águila, C. et al. Resistencia a insulin en adolescents obesos. An Fac Med, v.71, n.1, p.13-20, 2010.

- Samaan, S.H. Niveles de hormona estimulante de tiroides en niños obesos sin patologia tiroidea. Rev Horiz Med, v.14, n.4, p.23-28, 2012.

- Tams, T.R. Manual de gastroenterología en pequeños animales. $2^{\text {nd }} e d$. Buenos Aires: Inter-Médica, 2004. 438p.

- Veiga, A.P.; Price, C.A.; De Oliveira, S.T. et al. Association of canine obesity with reduced serum levels of C-reactive protein. J Vet Diagn Invest, v.20, n.2, p.224-228, 2008.

- Zentek, J.; Elices, R.; Goodar, F. et al. Obesidad en perros y gatos: Implicaciones dietéticas. Rev Canis et felis, v.106, p.6-19, 2010.

Cómo citar: Osorio, J.H.; Suárez, Y.J. Comparación del perfil lipídico y del perfil tiroideo en caninos adultos obesos vs. caninos adultos normales. Revista Veterinaria y Zootecnia, v.11, n.2, p.13-23, 2017. DOI: 10.17151/vetzo.2017.11.2.2 